\title{
Primary Device Identifier
}

National Cancer Institute

\section{Source}

National Cancer Institute. Primary Device Identifier. NCI Thesaurus. Code C101722.

The main identifier for a device. 